\title{
Isolation of peroxisomes from dental pulps by sucrose density gradient centrifugation
}

\author{
Toshio Mori, Kazuhiko Sasaki, Masamichi Terashita \\ and Miyota Nagano \\ Department of Operative Dentistry (Chief: Prof. Miyota Nagano), \\ Kyushu Dental College, Kokura, Kitakyushu 803, Japan
}

[Accepted for publication: January 25, 1985]

Key words: dental pulp/peroxisomes/microperoxisomes

\section{Introduction}

Since the discovery of fatty acid oxidation in rat liver peroxisomes, some interest has arisen as to the relative importance of peroxisomes $^{1)}$. Peroxisomes contain catalase which play an important role with superoxide dismutase and glutathione peroxidase in the defence mechanism against oxygen toxicity by active oxygen ${ }^{2}$.

Peroxisomes in oral tissues are also interesting organelles, because active oxygen has been described to be concerned with inflammation and tumors ${ }^{3,4}$. But there are no available literature data on isolation and properties of peroxisomes in animal oral tissues.

The purpose of the present study is to isolate the peroxisomes from mammalian dental pulp by sucrose density gradient centrifugation.

\section{Materials and Methods}

Dental pulps of rats (donryu strain), bovine, rabbits (Japanese white strain) and guinea pigs (Hartley strain) were used in this study.

Subsequent manipulations were carried out at $0-4^{\circ} \mathrm{C}$. Dental pulps were cut into small pieces with scissors and homogenized with 4 volumes of $0.25 \mathrm{M}$ sucrose $/ 20 \mathrm{mM}$ glycylglycine $(\mathrm{pH} 7.5)$ by one pass in a Potter-Elvehjem homogenizer with a Teflon pestle at a $1000 \mathrm{rev} . / \mathrm{min}$. The homogenate was filtered through one layer of cheese-cloth and centrifuged at $300 \times g$ for $5 \mathrm{~min}$ to remove cell debris. A portion $(2 \mathrm{ml})$ of the homogenate was layered on a $33 \mathrm{ml}$ linear sucrose density gradient $(26-60 \%, \mathrm{w} / \mathrm{v})$ and centrifuged at $132,400 \times g$ for $80 \mathrm{~min}$ in a Hitachi 55P-72 centrifuge (RPV 50-160). Fractions $(2.5 \mathrm{ml})$ were collected from the bottom of the tube.

Catalase $^{5)}$, glutamate dehydrogenase ${ }^{6)}$ and lactate dehydrogenase ${ }^{7)}$ were assayed by the methods cited. A unit of enzyme activity is defined as the amount of enzyme that catalyzes a formation of product or a decrease in substrate of $1 \mu \mathrm{mol} / \mathrm{min}$ at $37^{\circ} \mathrm{C}$.

\section{Results and Discussion}

Representative sedimentation of rat dental pulp in a sucrose density gradient centrifugation is shown in Fig. 1. The peroxisomes, identified by catalase marker, were obtained at a density of about $1.17 \mathrm{~g} / \mathrm{ml}$ and the mitochondria, identified by glutamate dehydrogenase marker, at a density of about $1.19 \mathrm{~g} / \mathrm{ml}$. Densities of two organelles differ from those in rat liver; in the liver, the density of peroxisomes is higher than that of mitochondria ${ }^{8}$. The two organelles showed different sedimentation profiles from other organelles such as lysosomes and microsomes (not shown).

A representative sedimentation for bovine and rabbit dental pulps in a sucrose density gradient centrifugation is shown in Figs. 2 and 3. Sedimentation profiles of catalase and 


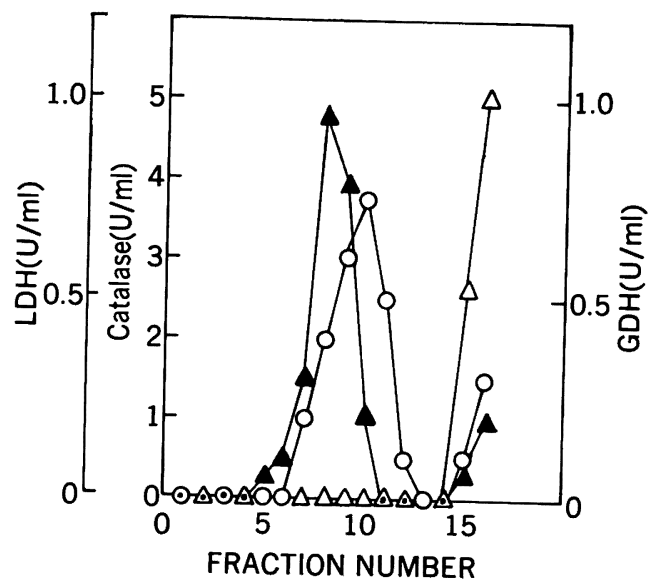

Fig. 1 Subcellular distribution of catalase, glutamaye dehydrogenase and lactate dehydrogenase in rat dental pulp. The postnuclear supernatant was prepared from rat dental pulp and subjected to sucrose density gradient centrifugation as described in the text: fractions of $2.5 \mathrm{ml}$ were collected. Catalase (O), gluamate dehydrogenase $(\mathrm{GDH}$, $\Delta)$ and lactate dehydrogenase $(\mathrm{LDH}$, $\Delta)$.

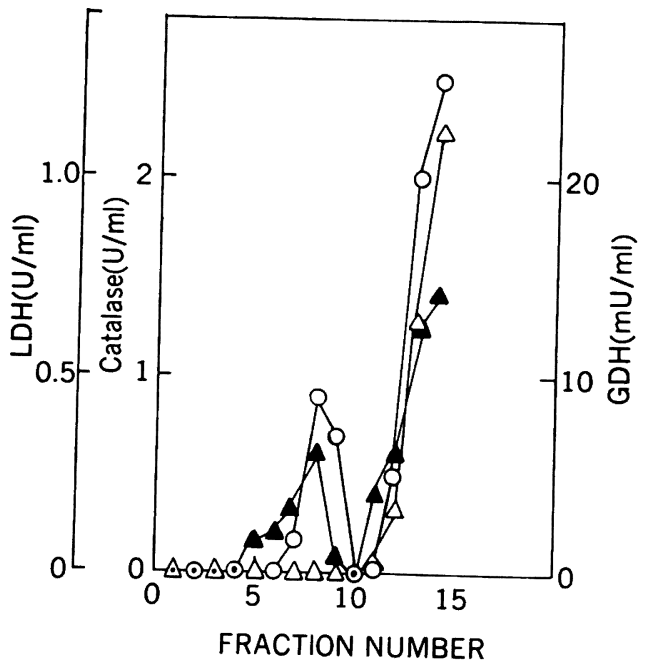

Fig. 2 Subcellular distribution of catalase, glutamate dehydrogenase and lactate dehydrogenase in bovine dental pulp. The postnuclear supernatant was prepared from bovine dental pulp and subjected to sucrose density gradient centrifugation as described in the text: fractions of $2.5 \mathrm{ml}$ were collected. Catalase (O), glutamate dehydrogenase $(\mathrm{GDH}, \boldsymbol{\Delta})$ and lactate dehydrogenase $(\mathrm{LDH}, \Delta)$.

glutamate dehydrogenase were nearly identical, suggesting that the peroxisomes were nearly identical with the mitochondria in the density. The two organelles were also not separated with the dental pulp of adult guinea pig and the dental papilla of fetal guinea pig (Fig. 4).

In the present study, peroxisomes were separated from other organelles with rat dental pulp. In rat dental pulp, the density 


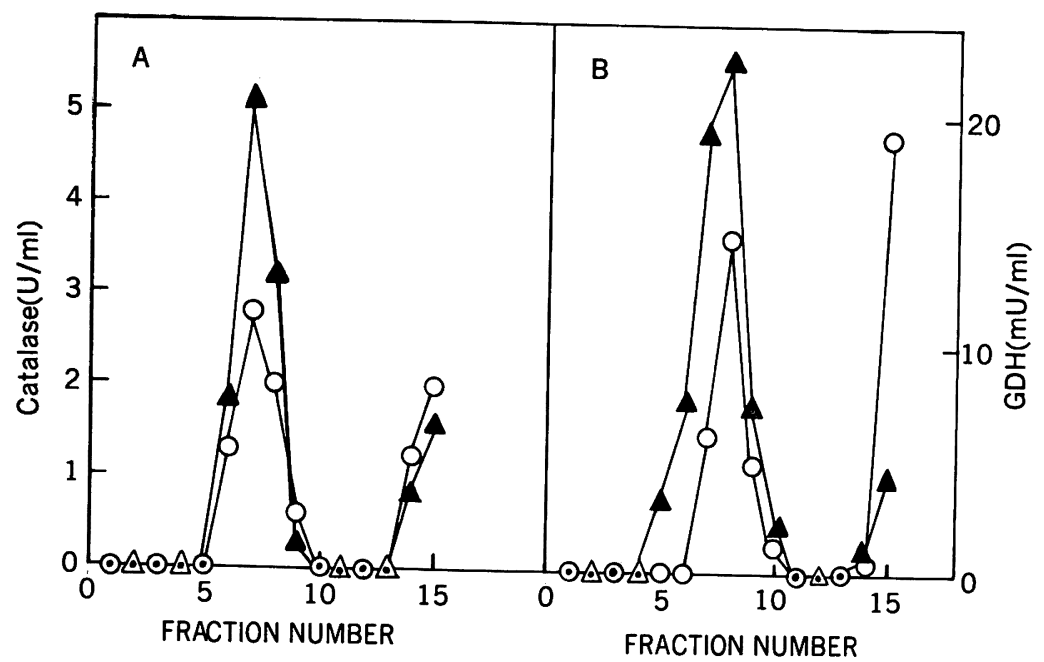

Fig. 4 Subcellular distribution of catalase and glutamate dehydrognase in adult guinea pig dental pulp (A) and fetal guinea pig dental papilla (B). The postnuclear supernatants were prepared from guinea pig dental pulp and dental papilla and subjected to sucrose density gradient centrifugation as described in the text: fractions of $2.5 \mathrm{ml}$ were collected. Catalase (O) and glutamate dehydrogenase (GDH, A).

of the peroxisomes was smaller than that of mitochondria, suggesting that the peroxisomes are so-called microperoxisomes ${ }^{9,10}$ ) which differ from the peroxisomes in morphology, but are similar in function. Studies on isolation of peroxisomes from dental pulps of bovine, rabbit and guinea pig are in progress.

\section{Acknowledgement}

We are grateful to Prof. Tomoo Noguchi, Department of Biochemistry, Kyushu Dental College, for his valuable advice. This study was supported in part by a Grant-in-Aid for Scientific Research from the Ministry of Education, Science and Culture, Japan (No. 59771414 in 1984).

\section{References}

1) Lazarow, P. B. and de Duve, C.: A fatty acyl-CoA oxidizing system in rat liver peroxisomes; enhancement by clofibrate a hypolipidemic drug. Proc. Natl. Acad. Sci. USA 73: 2043-2046, 1976.

2) Fridovich, I.: The biology of oxygen radicals. Science (Wash. DC) 201: 875-880, 1978.
3) McCord, J. M.: Free radicals and inflammation. Science 185: 529-531, 1974.

4) Floyd, R. A., Soong, L. M., Stuart, M. A. and Reigh, D. L.: Free radicals and carcinogenesis. Arch. Biochem. Biophys. 185: 450-457, 1978.

5) Sinha, A.K.: Colorrimetric assay of catalase. Anal. Biochem. 47: 389-394, 1972.

6) Beaufay, H., Bendall, D., Baudhuin, P. and de Duve, C.: Tissue fractionation studies, 12. Intracellular distribution of some dehydrogenase, alkaline deoxyribonuclease and iron in rat liver tissue. Biochem. J. 73: 623-628, 1959.

7) Reeves, W. J. and Figmonari, G. M.: Lactate dehydrogenase. Methods. Enzymol. 9: 288-290, 1966.

8) Schneider, W. G. and Hogeboom, G. H.: Intracellular distribution of cytochrome $\mathrm{c}$ in rat liver homogenates. J. Biol. Chem. 183: $123,1950$.

9) Novikoff, P. M. and Novikoff, A. B.: Peroxisomes in absorptive cells of mammalian small intestine. J. Cell. Biol. 53: 532-560, 1950.

10) Novikoff, A. B. and Novikoff, P. M.: Microperoxisomes. J. Histochem. Cytochem. 21 : 963-966, 1973. 